REVISTA CIENCIAS BIOMÉDICAS

PRESENTACIÓN DE CASOS CLÍNICOS

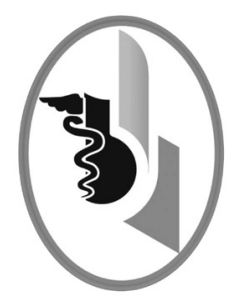

\title{
OBSTRUCCIÓN INTESTINAL INTERMITENTE SECUNDARIA A MALROTACIÓN EN LACTANTE MENOR
}

\author{
SECONDARY INTERMITTENT INTESTINAL OBSTRUCTION \\ TO MALROTATION IN AN INFANT: CASE REPORT
}

San Juan-Marconi Martha Karina ${ }^{1}$

Martínez-Romero Eliana ${ }^{1}$

De Vivero-Camacho Rodrigo ${ }^{2}$

Muñoz-Álvarez Nelson²

Correspondencia: eliana_mmr@hotmail.com

Recibido para evaluación: abril-25-2015. Aceptado para publicación: junio-24-2016

\section{RESUMEN}

Introducción: el vómito bilioso en el recién nacido requiere atención especial, dado que puede ser un síntoma secundario de patologías potencialmente graves que causan importante morbimortalidad en este grupo etario.

Caso clínico: se presenta el caso de un paciente lactante menor de un mes y once días de edad, quien presentó vómitos biliosos intermitentes desde el nacimiento, con varios ingresos hospitalarios sin mejoría. Cuando ingresó a la urgencia se consideró clínicamente la posibilidad la malrotación intestinal, por ello fue llevado a cirugía. Demostrada la alteración, y se realizó corrección con procedimiento de Ladd. La evolución del paciente fue satisfactoria pese al retraso en el diagnóstico.

Conclusión: el vómito bilioso en neonatos es una emergencia quirúrgica. Requiere la sospecha diagnóstica y realización de estudios dirigidos a establecer el diagnóstico etiológico. Rev.cienc.biomed. 2016;7(2):296-300.

PALABRAS CLAVE

Obstrucción intestinal; Lactante; Vómitos; Mortalidad infantil.

\section{SUMMARY}

Introduction: the bilious vomiting in newborns requires special attention because it can be a secondary symptom of potential grave pathologies that cause morbidity and mortality in this age group.

1 Médico. Estudiante de Postgrado. Pediatría. Facultad de Medicina. Universidad de Cartagena. Colombia.

2 Médico. Especialista en Pediatría. Docente. Departamento de Pediatría. Facultad de Medicina. Universidad de Cartagena. Colombia. 
Case report: this article is about an infant patient less than one month and 11 days of age, who presented intermittent bilious vomiting since birth with several hospital admissions for this reason without improvement. Then, intestinal malrotation was consider as a possible diagnosis when the patient was admitted in the hospital. After that, he was taken to surgery and was treated with Ladd procedure. The evolution of the patient was satisfactory despite delay in the diagnosis. It was carried out a literature review.

Conclusion: bilious vomiting in infants is a surgical emergency. It requires diagnostic suspicion and the implementation of more studies to establish the etiological diagnosis. Rev.cienc.biomed. 2016;7(2):296-300.

\section{KEYWORDS}

Intestinal obstruction; Infant; Vomiting; Infant mortality.

\section{INTRODUCCIÓN}

Los vómitos biliosos en neonatos son un signo inicial de obstrucción intestinal, por lo que deben ser considerados como una emergencia quirúrgica hasta que se demuestre lo contrario (1). La incidencia de emesis biliosa indicativa de obstrucción quirúrgica es reportada entre el 20 y $38 \%$ de los casos (2). Dentro de las causas se encuentran: atresia duodenal, malrotación intestinal, vólvulo, atresia yeyuno-ileal, íleo meconial y enterocolitis necrotizante (3). Todas estas entidades tienen en común el aumento de la morbilidad relacionada con desequilibrio hidroelectrolítico, hipoglicemia e isquemia intestinal, con consiguiente aumento de la mortalidad, principalmente asociado a los retrasos en el diagnóstico y el manejo específico (4). Un diagnóstico oportuno y acertado es la clave para el manejo satisfactorio (5).

\section{CASO CLÍNICO}

Se presenta el caso de un paciente masculino de un mes y once días de vida, nacido por cesárea programada que pesó al nacer 3600 gramos, sin antecedente de reanimación neonatal, que ingresó al servicio de urgencias por cuadro de vómitos biliosos intermitentes desde el nacimiento, asociados a pobre ganancia de peso (340 gramos) y múltiples consultas a instituciones de salud. A la llegada a urgencias no presentaba signos de deshidratación ni distensión abdominal. Se ordenó inmediatamente tránsito intestinal, que inicialmente fue interpretado normal (Figura No 1).

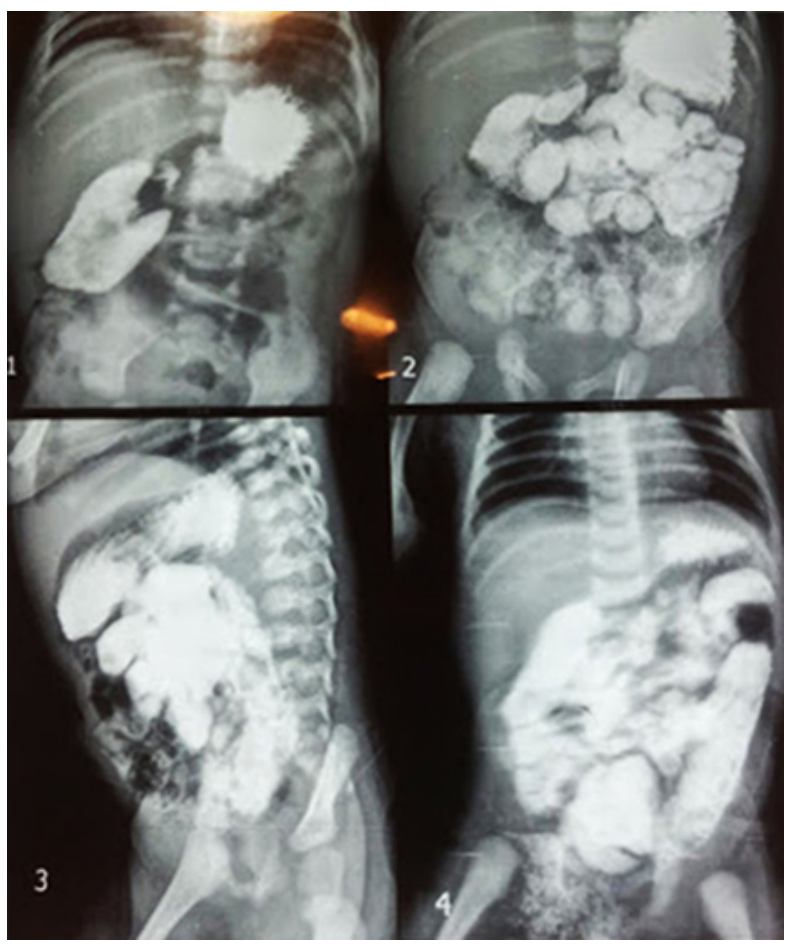

Figura No 1. Tránsito gastrointestinal al ingreso

Se revisaron las imágenes del tránsito intestinal por integrantes de los servicios de cirugía pediátrica y gastroenterología pediátrica, considerándose clínicamente la posibilidad de malrotación intestinal por observar duodeno elongado. El paciente fue sometido a laparotomía exploratoria; los hallazgos demostraron duodeno y estómago distendidos con ciego y apéndice en el cuadrante superior izquierdo fijados por bandas de Ladd (la técnica utilizada fue el procedimiento de Ladd). Los cuidados postoperatorios incluyeron monitorización 

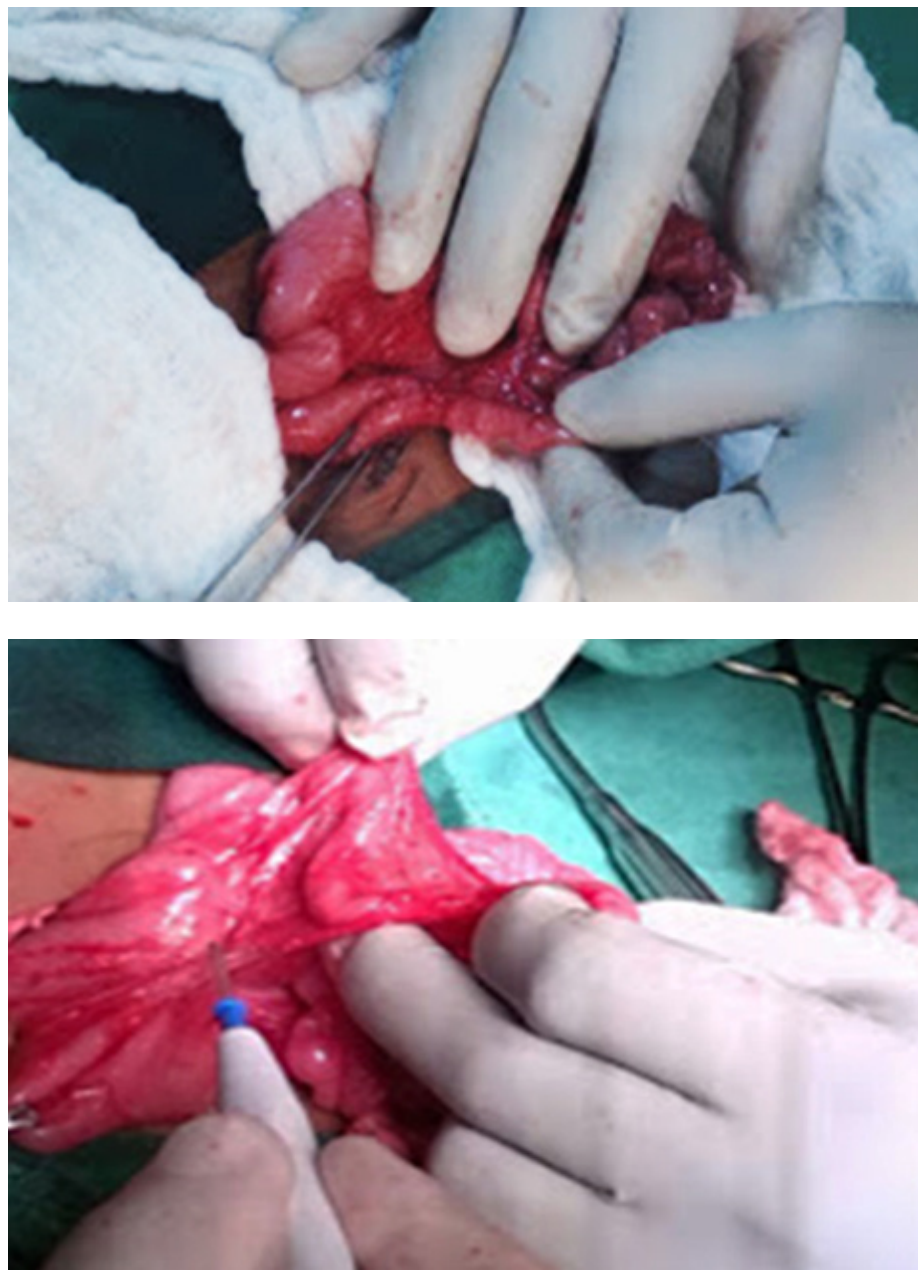

Figura $N^{\circ} 2$.

Detalles de la intervención operatoria

en unidad de cuidados intensivos. La evolución fue satisfactoria, adecuada tolerancia de la vía oral, sin complicaciones, por lo cual se dio de alta. Figura No 2.

\section{DISCUSIÓN}

La malrotación intestinal hace referencia a anormalidades en la posición y fijación del intestino. Se presenta en aproximadamente 1 de cada 500 nacimientos $(6,7)$, pero la incidencia de casos sintomáticos es calculada en 1: 6000 nacimientos (8). Usualmente se diagnostica en recién nacidos y lactantes menores; más del $75 \%$ de los casos sintomáticos ocurren en recién nacidos y más del $90 \%$ en el primer año de vida (9).

Nasir et al. (4) llevaron a cabo un estudio retrospectivo, incluyendo nueve pacientes (ocho niños y una niña) con diagnóstico de malrotación intestinal, con una edad media de presentación de 15 días. Existe un predominio de esta entidad en los pacientes de sexo masculino (10).

La manifestación clínica inicial de malrotación intestinal en neonatos es el vómito bilioso con o sin distensión abdominal. Otros síntomas descritos son dolor, irritabilidad, náusea y anorexia (11). En un estudio retrospectivo se evaluaron presentaciones de malrotación intestinal en niños, se encontró anorexia o náuseas en el $36 \%$, apnea intermitente en el $24 \%$ y falla de medro en el $41 \%$ (12). En otro estudio se encontraron variaciones en el patrón de las heces y diarrea en el $16 \%$ de los casos (10). La complicación más temida es el vólvulo de intestino medio, condición que amenaza la vida al comprometer el flujo sanguíneo de la arteria mesentérica superior, y se presenta comúnmente en el primer año de vida (13), en aproximadamente $70-90 \%$ de los neonatos con malrotación, con necrosis intestinal en cerca del $15 \%$ de los afectados.

Existen reportes en la literatura de formas de presentación atípicas para la malrotación intestinal, especialmente en niños mayores de un año. Zellos et al. (14) reportaron el caso de un paciente de 17 meses de edad con enteropatía con pérdida de proteínas secundaria por malrotación intestinal y vólvulo de intestino medio crónico que generó obstrucción linfática y derrame de linfa en el intestino y peritoneo.

Malhotra et al. (2) realizaron un estudio retrospectivo donde analizaron neonatos ingresados por vómitos biliosos. Encontraron 61 pacientes, de los cuales la mayoría correspondía a recién nacidos a término con peso adecuado para la edad gestacional. Dieciséis pacientes fueron sometidos a laparotomía, de los cuales seis presentaban malrotación intestinal con vólvulo. 
ISSN: 2215-7840, 7(2), julio-diciembre 2016, San Juan-Marconi Martha Karina, Martínez-Romero Eliana, De Vivero-Camacho Rodrigo,

Muñoz-Álvarez Nelson

El tránsito intestinal es el estudio de elección en todo niño que se presente con vómitos biliosos y debe ser realizado inmediatamente (15). Es un estudio relativamente no invasivo y generalmente disponible en centros pediátricos. Los hallazgos sugestivos son posición baja de la unión duodeno-yeyunal, ausencia de la unión duodeno-yeyunal en su posición anatómica típica a la izquierda del pedículo del cuerpo vertebral, yeyuno localizado a la derecha, duodeno redundante y apariencia en sacacorchos de la unión duodeno-yeyunal $(15,16)$. Puede tener algunas limitaciones, por ejemplo, la necesidad de mantener una posición adecuada durante todo el estudio, las imágenes se pueden distorsionar por distensión abdominal o sondas y la unión duodeno-yeyunal puede tener una variante normal de su posición (16).

Sizemore et al. realizaron un estudio retrospectivo (17), donde analizaron pacientes con sospecha de malrotación intestinal, a estos se les realizó tránsito intestinal, posteriormente fueron sometidos a procedimiento de Ladd. De 163 pacientes con diagnóstico confirmado en cirugía, 156 tuvieron un estudio positivo para malrotación (sensibilidad del 96\%); hubo dos pacientes con falsos positivos (posición yeyunal anormal) y siete con falsos negativos (seis con posición yeyunal normal).

Otros estudios habitualmente realizados en estos casos son la ecografía abdominal, enema con bario, TAC o RM abdominal, que no tienen la misma efectividad diagnóstica, son más costosos y menos accesibles (15).

El procedimiento de Ladd es el manejo de elección, ya sea abierto o laparoscópico con resultados similares (18). Esto implica desrotación del intestino si hay torsión, liberación de adherencias peritoneales, reposicionamiento de segmentos intestinales y apendicectomía (18). El retraso en el diagnóstico puede llevar a isquemia intestinal grave que requiera resección y anastomosis, con un aumento considerable de la morbimortalidad. Las complicaciones se han descrito hasta en el $46 \%$ de los pacientes, dentro de las más comunes se encuentran la obstrucción de intestino delgado y el síndrome de intestino corto, las cuales pueden requerir cirugía adicional (18).

En el estudio de Nasir et al. (4) encontraron una mortalidad global de $22 \%$ (dos pacientes), una de las muertes relacionada con isquemia intestinal masiva debida a vólvulo y fuga anastomótica, y la otra muerte asociada a obstrucción intestinal postoperatoria.

Se ha propuesto intervención quirúrgica diferida en varios tiempos, en los pacientes con isquemia intestinal extensa. Houben et al. (19) reportaron el caso de un paciente de veinte horas de vida con isquemia total de intestino delgado en laparotomía exploratoria inicial, sin mejoría a las 24 horas. Se ordenó reposo intestinal, sonda nasogástrica y nutrición parenteral durante once semanas; la laparotomía de control evidenció que cuarenta $\mathrm{cm}$ de intestino delgado estaban intactos.

Existen otras propuestas de manejo para limitar la extensión de la isquemia intestinal, con un tratamiento combinado, incluyendo masaje digital de vasos mesentéricos después de la desrotación seguida por infusión continua de activador tisular del plasminógeno. Kyely et al. (20) reportaron éxito en dos neonatos con esta técnica, aplicando el activador tisular del plasminógeno a las seis horas del procedimiento quirúrgico, durante 18 horas, con evidencia de resolución total de la isquemia intestinal en la laparotomía exploratoria de control.

\section{CONCLUSIÓN}

El vómito bilioso en neonatos es una emergencia quirúrgica. Requiere la sospecha diagnóstica y realización de estudios dirigidos a establecer el diagnóstico etiológico, descartando patologías potencialmente graves que amenazan la vida.

CONFLICTO DE INTERESES: ninguno que declarar.

FINANCIACIÓN: recursos propios de los autores. Estudios de laboratorio, insumos hospitalarios, medicamentos y honorarios profesionales aportados dentro de la atención asistencial. 


\section{REFERENCIAS BIBLIOGRÁFICAS}

1. Kimura K, Loening-Baucke V. Bilious vomiting in the newborn: rapid diagnosis of intestinal obstruction. Am Fam Physician. 2000;61(9):2791-2798.

2. Malhotra A, Lakkundi A, Carse E. Bilious vomiting in the newborn: 6 years data from a level III centre. J Paediatr Child Health. 2010;46(5):259-261.

3. Chandran L, Chitkara M. Vomiting in children: reassurance, red flag, or referral? Pediatr Rev. 2008;29:183-192.

4. Nasir AA, Abdur-Rahman LO, Adeniran JO. Outcomes of surgical treatment of malrotation in children. Afr J Paediatr Surg 2011;8:8-11.

5. Godbole $P$, Stringer MD. Bilious vomiting in the newborn: how offten is it pathologic? J. Pediatr Surg. 2002;37(6):909-911.

6. Ohshiro K, Puri P. Pathogenesis of infantile hypertrophic pyloric stenosis: recent progress. Pediatr Surg Int 1998, 13(4):243-52.

7. Williams H. Green for danger! Intestinal malrotation and volvulus. Arch Dis Child Educ Pract Ed. 2007;92:87-91.

8. Ingue R, Lange P. The Ladd's procedure for correction of intestinal malrotation with volvulus in children. Aorn J. 2007;85:300-308.

9. Applegate $\mathrm{KE}$, Anderson JM, Klatte EC. Intestinal malrotation in children: a problem-solving approach to the upper gastrointestinal series 1 . Radiographics 2006;26(5):1485-1500.

10. Millar AJ, Rode $\mathrm{H}$, Cywes S. Malrotation and volvulus in infancy and childhood. Semin Pediatr Surg 2003;12:229-236.

11. Shalaby MS, Kuti K, Walker G. Intestinal malrotation and volvulus in infants and Children. BMJ. 2013;347:6949.

12. Powell DM, Othersen HB, Smith CD. Malrotation of the intestines in children: the effect of age on presentation and therapy. J Pediatr Surg. 1989;124:777-780.

13. Strouse PJ: Disorders of intestinal rotation and fixation ("malrotation"). Pediatr Radiol 2004;34(11):837-851.

14. Zellos A, Diagoras Zarganis D, Ypsiladis S, et al. Malrotation of the intestine and chronic volvulus as a cause of protein-losing enteropathy in infancy. Pediatrics. 2012;129:e515-e518.

15. Tackett J, Muise E, Cowler R. Malrotation: current strategies navigating the radiologic diagnosis of a surgical emergency. World J Radiol. 2014;6(9): 730-736.

16. Maxfield $\mathrm{CM}$, Bartz BH, Shaffer JL. A pattern-based approach to bowel obstruction in the newborn. Pediatr Radiol. 2013;43:318-329.

17. Sizemore AW, Rabbani KZ, Ladd A, Applegate KE. Diagnostic performance of the upper gastrointestinal series in the evaluation of children with clinically suspected malrotation. Pediatr Radiol. 2008;38:518-528.

18. Mitsunaga $T$, Saito $T$, Terui $\mathrm{K}$, et al. Risk factors for intestinal obstruction after Ladd procedure. Pediatric Reports. 2015;7:31-34.

19. Houben $\mathrm{CH}$, Mitton $\mathrm{S}$, Capps S. Malrotation volvulus in a neonate: a novel surgical approach. Pediat Surg Int. 2006; 22 (4): 393-394.

20. Kiely EM, Pierro A, Pierce C. Clot dissolution: a novel treatment of midgut volvulus. Pediatrics. 2012;129:e1601-e04.

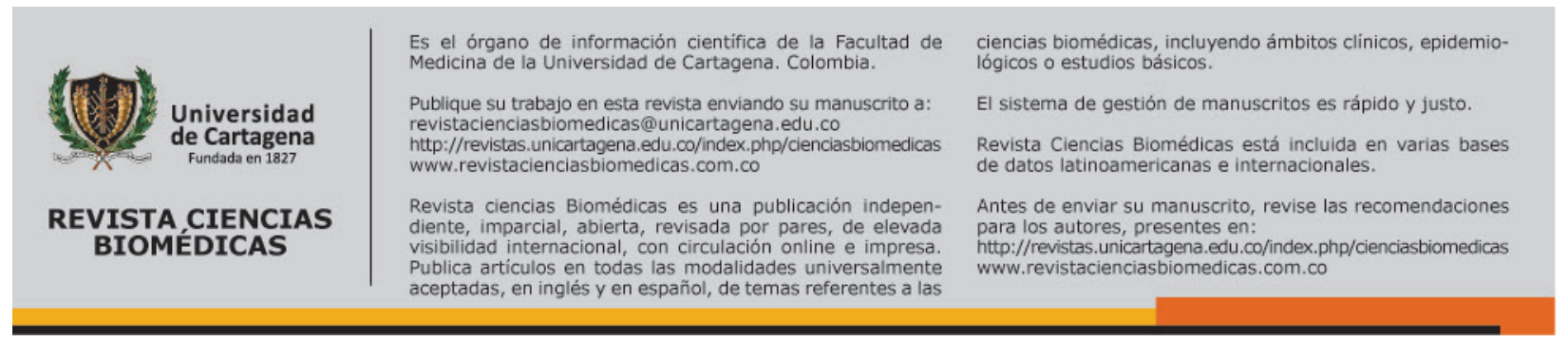

